those interested in some aspect of the electrochemistry of oxygen. The vast amount of material relevant to chapters two to five, concerned with the oxygen electrode at rest, the anodic evolution and cathodic reduction of oxygen on noble metals and the reversible oxygen clectrode, is well ordered and clearly and critically presented. Conflicting views on reaction mechanisms, the role of hydrogen peroxide and the nature and significance of oxides on platinum are given in a balanced manner. There are some omissions; there is, for example, no discussion of the information concerning the oxides on platinum available from studies of other reactions such as the Kolbé synthesis. These four chapters form the heart of the book and the next, closely related to them, is a useful discussion on the electroanalytical chemistry of oxygen. Sections describing the oxygen electrode on mercury and the reduction of oxygen in non-aqueous systems also appear in this chapter.

The initial coherence and clear purpose are somewhat diminished in the remaining three chapters and lead one to question the concept of bringing together in a single volume diverse topics linked only by the common involvement of oxygen in an electrochemical situation. The oxyger eloctrode on active metals, porous oxygen electrodes and the role of oxygen in corrosion mechanisms are discussed as particular examples of the applications of oxygen electrodes. The long chapter on active metals is mainly concerned with the properties of oxide electrodes used in batteries and is restricted to silver, lead and nickel. Valve metals and semiconductors are specifically excluded from the scope of the book. Inevitably, restricting the discussion of this broad topic to a single chapter leaves no room for the consideration of many important aspects. The section on porous oxygen electrodes is a condensed summary of a cross-section of the field ranging from basic kinetic mechanisms to engineering aspects and is a useful source of references. A chapter on the role of oxygen in corrosion mechanisms brings the book rather uneasily to an end. Although within the space of thirty pages a summary is given of a surprisingly large number of aspects of corrosion, the result highlights the difficulty of treating such a subject in a short space at the standard set by the first half of the book.

The book is always very readable in spite of covering a tremendous amount of material. It will be widely appreciated by almost all varieties of electrochemist, but mainly for the contents of the first half.

\section{A. BewtCK}

\section{OPERATORS FOR PHYSICISTS}

\section{Induced Representations of Groups and Quantum Mechanics}

By George W. Mackey. (Publicazioni della Classe di Scienze.) Pp. viii +167 . (Benjamin: New York and Amsterdam; Editora Boringhieri: Torino, 1968.) \$8.50. Since the early days of the quantum theory there has been much discussion concerning the concept of the localizability of a particle and thus of the operator corresponding to the position of a particle. From the point of view of the quantum theory of measurement and of relativistic field theory these questions are still open, but for non-relativistic quantum mechanics and relativistic single-particle quantum mechanics there are now rigorous mathematical proofs available for statements such as: "The photon is not localizable and neither is a neutrino of specific helicity."

In quantum mechanies, the operators describing the property for a particle that it may be localized in a region $S$ of physical space are projection operators $P_{S}$ which operate in the Hilbert space of states and obey the five axioms: (1) To every reasonable (that is, Borel) set $S$ there is a projection operator $P_{S}$ whose expectation value is the probability of finding the particle in $S$; (2) $P$ (common part of two sets $S_{1}, S_{2}$ ) $=P_{S_{1}} \cdot P_{S_{2}}$; (3) $P$ (union of two disjoint sets $\left.S_{1}, S_{2}\right)=P_{S_{1}}+P_{S_{2}}$; (4) $P$ (all space) $=1$; (5) $P$ (region obtained from $S$ by rotation and translation $)=U P_{S} U^{-1}$, where $U$ is the unitary operator on wave functions whose application gives new wave functions obtainable from the old by the appropriate rotations and translations.

That a particle localized in both $S_{1}$ and $S_{2}$ is localized in $S_{1} \cap S_{2}$, that a particle localized in $S_{1} \smile S_{2}$ is localized in $S_{1}$ or $S_{2}$ and that a particle is somewhere, are clearly intuitive. These statements form the basis of axioms (1) to (4). The heart of the theory of localizable states lies in axiom (5). Professor Mackey has developed a theory of systems of imprimivity and of indueed representations of a topological group $G$ tailored for these axioms. The theory goes beyond the above application to localizability in which $G$ is the group of translations and rotations in space. This book expounds this theory and is based on lectures given at the Scuola Normale, Pisa, in 1967. If $g \& G$ and $G$ has two realizations, one by homeomorphisms $T(g)$ of a topological space $\mathrm{E}, x \rightarrow T(g) x$, and one by unitary transformations $U(g)$ in a Hilbert space $\mathcal{H}$, the generalization of axiom (5) is

$$
P_{T_{(g) S}}=U(g) P_{S} U(g)^{-1}
$$

the $P$ s forming a system of imprimivity in $H$. 'The basic theorem states that for transitive $T$ (where each point $x$ in $\mathbf{E}$ is carried into any arbitrary point of $\mathbf{E}$ by a suitable $T(g)$ ) the projection operators and unitary operators can be brought into standard form. This standard form is what Mackey has called the induced representation associated with a unitary representation of a subgroup of $G$ having a fixed point in E.

This book is not easy for physicists to read. They will learn that what they have been doing for forty years, namely, writing the Schrödinger representation operators for $q, p$ and $H$, is correct and essentially unique. They will learn that von Neumann's proof concerning the uniqueness of irreducible representations for the Heisenberg canonical commutation relations has withstood the probing and generalizing of the next generation of mathematicians. They will learn that the angular momentum really does break up into orbital and spin. They will probably dismiss the book as doing what in North America is called "nit-picking". There are some of us, however, who like to distinguish between that which has been proved from stated assumptions and that which is, so far, essentially the handwaving of our more exuberant brethren. This book is for us.

E. A. Power

\section{ALGEBRAIC STRUCTURES}

\section{Introduction aux Structures Algébriques}

By J. Levy-Bruhl. (Monographies Universitaires de Mathématiques, No. 32.) Pp. xiv +328 . (Dunod: Paris, 1968.) 76 francs.

"This book, based on an algebra course taught in the Science Faculty at Reims, does not require, in principle, any prior mathematical knowledge.... After recalling the definitions of classical algebraic structures, their common characteristics ... are described: the basic idea is that of an ordered algebraic structure, with an order relation compatible with the operation. Categories are presented in a purely algebraic manner ... " (Reviewer's translation.)

I must declare my conviction that, although it is strictly true that no prior mathematical knowledge is required for a "local" understanding of this text, enormous sophistication and mathematical experience are required for a global understanding of the material. The total absence of motivation is forbidding; the reader is 